\title{
Cell-to-cell communication via extracellular vesicles among human pancreatic cancer cells derived from the same patient
}

\author{
MITSURU CHIBA $^{1}$, SHIORI KUBOTA ${ }^{2}$, AYAKA SAKAI ${ }^{2}$ and SATORU MONZEN ${ }^{3}$ \\ Departments of ${ }^{1}$ Bioscience and Laboratory Medicine, ${ }^{2}$ Medical Technology and ${ }^{3}$ Radiation Science, \\ Graduate School of Health Sciences, Hirosaki University, Hirosaki, Aomori 036-8564, Japan
}

Received April 17,2018; Accepted August 7, 2018

DOI: $10.3892 / \mathrm{mmr} .2018 .9376$

\begin{abstract}
Despite existing multimodal therapies, pancreatic cancer exhibits high metastatic capability and poor prognosis. Extracellular vesicles (EVs) are nanoparticles comprising lipid bilayers and various other components, such as protein and nucleic acids, derived from secreted cells. Recent research has demonstrated the involvement of EVs released from cancer cells in the metastasis of cancer cells to distant organs. However, the effects of EVs released from pancreatic cancer cells on other pancreatic cancer cells in a tumor microenvironment remain unclear. The present study aimed to elucidate that EVs released from PK-45H pancreatic cancer cells are taken up by PK-45P pancreatic cancer cells derived from the same patient through dynamin-related endocytosis. Additionally, EVs released from PK-45H cells augment the phosphorylation of classical mitogen-activated protein kinase (MAPK) pathways in PK-45P cells. The uptake of EVs released from PK-45H cells by PK-45P cells stimulates cell migration through the classical MAPK-dependent pathway, suggesting that EVs released from one pancreatic cancer cell are taken up by other surrounding pancreatic cancer cells and could be critical inducers of cancer metastasis in the tumor microenvironment.
\end{abstract}

\section{Introduction}

Extracellular vesicles (EVs), which are small particles released by various cells into the extracellular environment (1), were first described by Pan and Johnstone from sheep reticulocytes in 1983 (2). EVs is a generic term, which includes exosomes, shedding microvesicles and apoptotic bodies (1). EVs with diameters ranging from 30 to $1,000 \mathrm{~nm}$ comprise a lipid bilayer membrane that encases the cytosol, as well as

Correspondence to: Dr Mitsuru Chiba, Department of Bioscience and Laboratory Medicine, Graduate School of Health Sciences, Hirosaki University, 66-1 Hon-cho, Hirosaki, Aomori 036-8564, Japan

E-mail: mchiba32@hirosaki-u.ac.jp

Key words: extracellular vesicles, pancreatic cancer, endocytosis, cell migration, ERK1/2 various components, including proteins (3-5), mRNAs (6), microRNAs (6-8), long noncoding RNAs (9), natural antisense RNAs (10), and DNAs (11). Subsequent studies have reported that the release of EVs is a crucial mediator of intercellular communication involved in normal physiological processes, as well as the pathological progression (12-14). Moreover, released EVs can exert their effects on recipient cells through various signaling pathways and trigger specific phenotypic changes (15-17).

It is imperative to comprehend the molecular mechanisms of the EV release from donor cells and the processes by which they are taken up by recipient cells. Previously, the biogenesis of exosome-like EVs has been suggested to be associated with the endosomal sorting complex required for transport (ESCRT) (18). Additionally, ESCRT-related proteins, such as ESCRT-I, -II, -III, and Alix, have been reported to be recruited to the cytosolic sides of multivesicular bodies (MVBs) to form exosome-like EVs (19). Neutral sphingomyelinase 2, a rate-limiting enzyme in ceramide biosynthesis, affects the secretion of microRNAs $(20,21)$. Nearby cells or reach distant cells uptake released EVs through the blood or other body fluids. Studies have suggested that the mechanisms of uptake include endocytic pathways, such as endocytosis, phagocytosis, micropinocytosis, and direct fusion with the cellular membrane (22-25).

Pancreatic cancer, most commonly known as pancreatic ductal adenocarcinoma (PDAC), remains one of the most deadly diseases with limited effective therapies available. Despite a better understanding of tumor biology and optimization of current treatment modalities, the 5-year survival rate of pancreatic cancer is only $8 \%$ in the United States (26). Perhaps, the low survival rate of PDAC could be attributed to an aggressive biological phenotype, which is characterized by early local invasion and metastasis (27-29). Thus, elucidating the molecular mechanisms associated with the occurrence, development, therapeutic resistance, and metastasis of this lethal disease is a priority. The invasion and metastasis of tumors, such as PDAC, are complicated processes involving several extracellular factors. Some recent studies reported the involvement of EVs released from pancreatic cancer in cell-to-cell communication among various cells, such as endothelial (30), stellate (31), fibroblast $(32)$, and immune $(33,34)$ cells, under a tumor microenvironment. Masamune et al reported that exosome-like EVs released from pancreatic cancer cells 
induced the AKT phosphorylation and mitogen-activated protein kinase (MAPK) signaling in pancreatic stellate cells (31). However, signaling and phenotypic alterations among pancreatic cancer cells through EV-induced endocrine or paracrine signaling remain unknown. Using several uptake inhibitors on pancreatic cancer cells obtained from the same patient, this study aims to investigate the uptake mechanisms of EVs released from pancreatic cancer cells and their effects on signaling and to examine whether EVs released from high-metastasis pancreatic cancer cells enhance the capacity of metastasis in low-metastasis pancreatic cancer cells.

\section{Materials and methods}

Cell culture. Riken BRC (Ibaraki, Japan) provided human pancreatic cancer cell lines PK-45H (RCB1973) and PK-45P (RCB2141) through the National Bio-Resource Project of the MEXT, Japan. PK-45H cells were derived from liver metastasis (high metastasis), whereas PK-45P cells were derived from a primary tumor (low metastasis) (35). Subsequently, we cultured PK-45H and PK-45P cells obtained from the same patient using RPMI-1640 medium (Wako, Tokyo, Japan) supplemented with $10 \%$ fetal bovine serum (Thermo Fisher Scientific, Inc., Waltham, MA, USA), $100 \mathrm{U} / \mathrm{ml}$ of penicillin, and $100 \mu \mathrm{g} / \mathrm{ml}$ of streptomycin (Wako) at $37^{\circ} \mathrm{C}$ in a humidified atmosphere of $5 \% \mathrm{CO}_{2}$.

Purification and detection of EVs. We plated PK-45H cells on $10 \mathrm{~cm}$ dishes at a density of $2 \times 10^{5}$ cells per dish in the culture media. Culture media were discarded after 4 days, dishes were washed thrice in a serum-free culture medium, and $10 \mathrm{ml}$ of the serum-free culture medium was added to each dish. Then, cell culture media were collected after $48 \mathrm{~h}$, and EVs from cell culture media were differentially centrifuged and purified using a previously reported procedure (10). After that, EV pellets were suspended in Dulbecco's phosphate-buffered saline [D-PBS (-)] or RPMI-1640 medium. We use a NanoSight LM10 instrument (NanoSight, Malvern, UK) to detect EVs released from PK-45H cells.

EV RNA isolation and detection. Total RNAs and EVs released from PK-45H cells were isolated using Isogen II (NipponGene, Tokyo, Japan). Then, we examined the sizes of extracted total RNAs using an Agilent 2100 Bioanalyzer and Agilent RNA 6000 Pico kits (Agilent Technologies, Inc., Santa Clara, CA, USA).

Uptake of EVs into cells. PK-45P cells were seeded into 8-well chamber slides at a density of $2 \times 10^{4}$ cells per well. After $24 \mathrm{~h}$, the slides were washed thrice in D-PBS (-). Then, we added RPMI-1640 containing $10 \mathrm{ng} / \mu 1$ of EVs stained with BODIPY TR Ceramide reagent or SYTO RNA Select reagent (both Thermo Fisher Scientific, Inc.) into each well with or without dynasore (Sigma-Aldrich; Merck KGaA, Darmstadt, Germany) of a dynamin-dependent endocytosis inhibitor, chlorpromazine (Sigma-Aldrich; Merck KGaA) of a clathrin-mediated endocytosis inhibitor, nystatin (Wako) of a caveolin-mediated endocytosis inhibitor, or cytochalasin D (Wako) of a micropinocytosis inhibitor. We incubated PK-45P cells for $3 \mathrm{~h}$ at $37^{\circ} \mathrm{C}$ in a humidified atmosphere of $5 \% \mathrm{CO}_{2}$. Then, PK-45P cells were treated with $4 \%$ paraformaldehyde solution at room temperature for $20 \mathrm{~min}$, followed by washing the slides thrice in D-PBS (-). After nuclei staining using ProLong Gold Antifade Reagent with 4',6-diamidino-2-phenylindole (DAPI; Thermo Fisher Scientific, Inc.), we covered the slides with coverslips and visualized using a confocal laser scanning microscope (LSM710; Carl Zeiss, Oberkochen, Germany) under similar conditions.

Immunofluorescence staining. PK-45P cells were plated at a density of $5 \times 10^{4}$ cells per well in 12-well plates with round coverslips. We added PK-45H EVs stained with SYTO RNA Select reagent to PK-45P cells and incubated for $3 \mathrm{~h}$ at $37^{\circ} \mathrm{C}$ in a humidified atmosphere of $5 \% \mathrm{CO}_{2}$. Then, the coverslips were washed thrice in TBS buffer $(25 \mathrm{mM}$ Tris- $\mathrm{HCl}$ and $150 \mathrm{mM} \mathrm{NaCl} ; \mathrm{pH}$ 7.2) and fixed with $4 \%$ paraformaldehyde solution. After that, the coverslips were washed thrice with TBS buffer and incubated with a membrane-permeating solution ( $0.2 \%$ Triton X-100 in TBS buffer) at room temperature for $5 \mathrm{~min}$. After washing thrice with TBS buffer, we incubated the coverslips with an Image-iT FX signal enhancer solution (Thermo Fisher Scientific, Inc.) at room temperature for $30 \mathrm{~min}$. Subsequently, the coverslips were incubated with Can Get Signal Immunostain Solution A (Toyobo, Osaka, Japan) containing a primary rabbit monoclonal antibody raised against lysosome-associated membrane protein 1 (LAMP1) (no. 9091, Cell Signaling Technology, Inc., Danvers, MA, USA) at a 1:200 dilution for $60 \mathrm{~min}$ at room temperature. Next, we washed the coverslips five times with TBS buffer and incubated at room temperature for $60 \mathrm{~min}$ with an anti-rabbit immunoglobulin $\mathrm{G}(\mathrm{IgG})$ Alexa Fluor 647-conjugated secondary antibody (no. 4414; Cell Signaling Technology, Inc.) prepared in Can Get Signal Immunostain Solution B at a 1:1,000 dilution. Then, the coverslips were washed five times with TBS buffer followed by nuclei staining using ProLong Gold Antifade Reagent with DAPI. Finally, we visualized stained cells using a confocal laser scanning microscope (LSM710; Carl Zeiss) under the same conditions.

Western blotting. PK-45P cells treated with or without EVs released from PK-45H cells were lysed using the M-PER Mammalian Protein Extraction Reagent (Thermo Fisher Scientific, Inc.) supplemented with Halt proteinase inhibitor cocktail (Thermo Fisher Scientific, Inc.). We measured protein concentrations using a BCA Protein Assay Kit (Thermo Fisher Scientific, Inc.) and a Benchmark Microplate Reader (Bio-Rad Laboratories, Inc., Hercules, CA, USA). Protein samples were prepared using $4 \mathrm{X}$ sample buffer $(0.25 \mathrm{~mol} / \mathrm{l}$ Tris- $\mathrm{HCl}, 8 \%$ SDS, $40 \%$ glycerol, $20 \%$ 2-mercaptoethanol, and $0.02 \%$ bromophenol blue; $\mathrm{pH} 6.8$ ). All samples were boiled at $100^{\circ} \mathrm{C}$ for $5 \mathrm{~min}$ and immediately cooled on ice. Then, we performed electrophoresis using 12\% Mini-Protean TGX Precast Gels (Bio-Rad Laboratories, Inc.) and electrophoresis buffer ( $25 \mathrm{mM}$ Tris- $\mathrm{HCl}, 192 \mathrm{mM}$ glycine, and $0.1 \%$ SDS; pH 8.3) at $200 \mathrm{~V}$ and $0.03 \mathrm{~A}$ for $30 \mathrm{~min}$. Proteins in gels were transferred to Trans-Blot Turbo Transfer Pack Mini PVDF membranes (Bio-Rad Laboratories, Inc.) using transfer buffer $(25 \mathrm{mM}$ Tris-HCl, $192 \mathrm{mM}$ glycine, $0.01 \%$ SDS, and 20\% methanol; pH 8.3) and a Trans-Blot Turbo 
Transfer System (Bio-Rad Laboratories, Inc.) at $25 \mathrm{~V}$ and 2.5 A for 3 min. After that, we washed PVDF membranes with TBST buffer $(25 \mathrm{mM}$ Tris- $\mathrm{HCl}, 150 \mathrm{mM} \mathrm{NaCl}$, and $0.05 \%$ Tween-20; pH 7.2) and incubated in PVDF Blocking Reagent for Can Get Signal (Toyobo) at room temperature for $60 \mathrm{~min}$. After blocking, we incubated membranes in Can Get Signal Immunoreaction Enhancer Solution 1 (Toyobo) containing the indicated primary antibody at room temperature for $60 \mathrm{~min}$. In this study, the primary antibodies used were as follows: Anti-GAPDH rabbit polyclonal antibody (no. ab9485; Abcam, Cambridge, UK), anti-phospho-c-RAF (Ser338; 56A6) rabbit monoclonal antibody (no. 9427; Cell Signaling Technology, Inc.), anti-phospho-MEK1/2 (Ser217/221; 41G9) rabbit monoclonal antibody (no. 9154; Cell Signaling Technology, Inc.), anti-phospho-p90RSK (Ser380; 9D9) rabbit monoclonal antibody (no. 9335; Cell Signaling Technology, Inc.), anti-phospho-ERK1/2 (Thr202/Tyr204) rabbit monoclonal antibody (no. 4370; Cell Signaling Technology, Inc.), and anti-ERK1/2 (137F5) rabbit monoclonal antibody (no. 4695; Cell Signaling Technology, Inc.). Membranes were washed five times for $5 \mathrm{~min}$ with TBST buffer and incubated at room temperature for $60 \mathrm{~min}$ with an anti-rabbit IgG horseradish peroxidase (HRP)-linked antibody (no. 7074; Cell Signaling Technology, Inc.) prepared in Can Get Signal Immunoreaction Enhancer Solution 2 (Toyobo). Then, we washed membranes five times for $5 \mathrm{~min}$ with TBST buffer and visualized bound antibodies using an ImmunoStar Zeta chemiluminescence system (Wako). Finally, images were analyzed using ChemiDoc XRS (BioRad Laboratories, Inc.) with Quantity One software (Bio-Rad Laboratories, Inc.).

Wound-healing assay. PK-45P cells were seeded at a density of $1 \times 10^{5}$ cells per well in 24-well flat-bottomed microplates and cultured to $90 \%$ confluency at $37^{\circ} \mathrm{C}$ in a humidified atmosphere of $5 \% \mathrm{CO}_{2}$. A thin scratch (wound) was made in the central area using pipette tips, following which cells were carefully washed thrice with D-PBS (-). Then, RPMI-1640 containing $10 \mathrm{ng} / \mu \mathrm{l}$ of EVs released from PK-45H cells was added to scratched PK-45P cells. We measured the width of wound closure under an IX71 light microscope (Olympus, Tokyo, Japan) using DP2-BSW software (Olympus). All images were acquired at $0,6,12,24,30$, and $36 \mathrm{~h}$ post-scratching.

Statistical analysis. In this study, statistical analysis was performed using Excel 2010 (Microsoft Corporation, Redmond, WA, USA) with the add-in software Statcel 3 (OMS Publishing Inc., Saitama, Japan). $\mathrm{P}<0.05$ was considered to indicate a statistically significant difference. One-way ANOVA was performed followed by Tukey's multiple comparisons test.

\section{Results}

Detection of EVs released from pancreatic cancer cells. We used human pancreatic cancer cell line $\mathrm{PK}-45 \mathrm{H}$ for the collection of EVs into culture media, as described in the Materials and methods section. Using the NanoSight LM10 instrument (NanoSight), we examined the size and amount of EVs released from PK-45H cells. The detected EV particles had a diameter of $126 \pm 7.6 \mathrm{~nm}$ (Fig. 1A). We extracted EV RNAs using Isogen II reagent (NipponGene) to confirm whether EVs released from PK-45H cells contained RNAs. Additionally, the size of the cellular and EV RNAs was examined using the Agilent 2100 Bioanalyzer (Agilent Technologies, Inc.,). We detected peaks for 18 and 28S ribosomal RNAs (rRNAs) in cellular RNAs of PK-45H. Also, we detected a single small RNA peak (size range: 25-200 nucleotides) in extracted EV RNAs but not rRNA peaks (Fig. 1B). These findings suggested that EVs released from $\mathrm{PK}-45 \mathrm{H}$ cells exhibited exosomal characteristics.

Uptake of EVs released from pancreatic cancer cells by other pancreatic cancer cells via dynamin-related endocytosis. Reportedly, EV uptake occurs through clathrin-mediated endocytosis, caveolin-mediated endocytosis, or micropinocytosis (36). However, the mechanism through which EVs released from pancreatic cancer cells are taken up by other pancreatic cancer cells remains unclear. As such, we assessed the recipient pancreatic cancer cell uptake of EVs labeled with fluorescent dyes using various endocytosis inhibitors. Then, we added $10 \mathrm{ng} / \mu \mathrm{l}$ of EVs stained with BODIPY TR Ceramide reagent or SYTO RNA Select reagent (Thermo Fisher Scientific, Inc.) to culture media of PK-45P cells treated with dynasore of a dynamin-dependent endocytosis inhibitor, chlorpromazine of a clathrin-mediated endocytosis inhibitor, nystatin of a caveolin-mediated endocytosis inhibitor, or cytochalasin D of a micropinocytosis inhibitor to investigate PK-45P pancreatic cancer cell uptake of EVs released from PK-45H cells. Accordingly, PK-45P cell fluorescence declined when EVs were added to PK-45P cells treated with $50 \mu \mathrm{M}$ chlorpromazine, $50 \mu \mathrm{M}$ nystatin, or $1 \mu \mathrm{M}$ dynasore (Fig. 2A-C). In contrast, we did not observe the EV uptake in PK-45P cells treated with up to $5 \mu \mathrm{M}$ cytochalasin D (Fig. 2D). These findings suggested dynamin-related endocytosis, such as clathrin- and caveolin-mediated endocytosis, and not micropinocytosis, as the mechanism through which EVs released from pancreatic cancer cells may be taken up by other pancreatic cancer cells.

EV RNAs taken up by PK-45P cells are not colocalized in lysosomes. EVs released from donor cells are taken up by recipient cells and trigger changes in various functions. We analyzed the colocalization of EV RNAs in lysosomes to determine whether EV components taken up by PK-45P cells exhibited any effect. Additionally, EV RNAs released from PK-45H cells were stained with SYTO RNA Select reagent (Thermo Fisher Scientific, Inc.) and added to culture media of PK-45P cells, as described in the Materials and methods section. Then, we used fluorescence immunostaining to detect LAMP1, a lysosome marker, in PK-45P cells. Accordingly, EV RNAs taken up by PK-45P cells were not colocalized in lysosomes (Fig. 3); this crucial result suggested that several EV components released from pancreatic cancer cells do not degrade and function in other pancreatic cancer cells.

Enhanced PK-45P cell migration through EVs released from $P K-45 H$ cells via activation of classical MAPK pathways. Reportedly, EVs released from cancer cells transfer information of donor cells (6). Qu et al reported that EVs released from cancer cells enhanced the phosphorylation of classical MAPK 

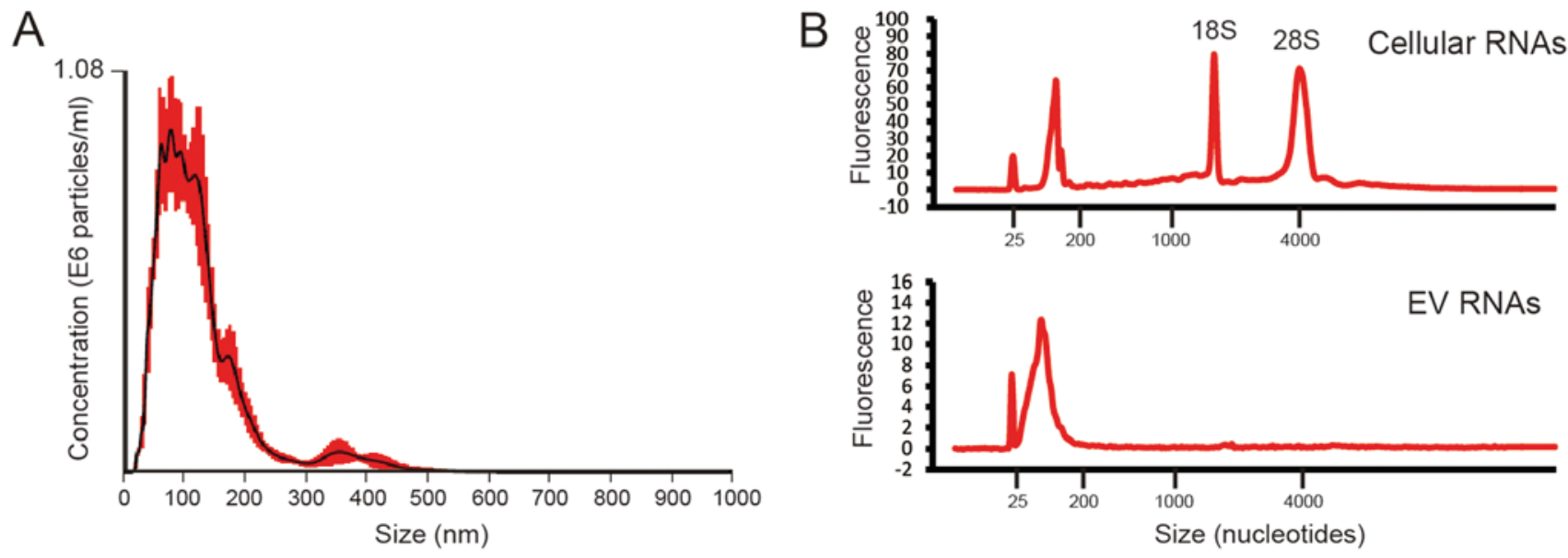

Figure 1. Detection of EV particles and small RNAs. (A) The detection of EV particles released from PK-45H cells using the NanoSight LM10 instrument. Red bar, the standard errors of the means; horizontal axis, the particle size (nm); vertical axis, the particle concentration (x $10^{6}$ particles $\left./ \mathrm{ml}\right)$. (B) The detection of EV RNAs released from PK-45H cells and cellular RNAs using the Agilent 2100 Bioanalyzer and Agilent RNA 6000 Pico kit. The peak detected 25 nucleotides (nt), representing an internal standard. The peak of small RNAs in the 25-200 nt size range was detected in EV RNAs. The experiments were performed twice to confirm reproducibility. EV, extracellular vesicle.
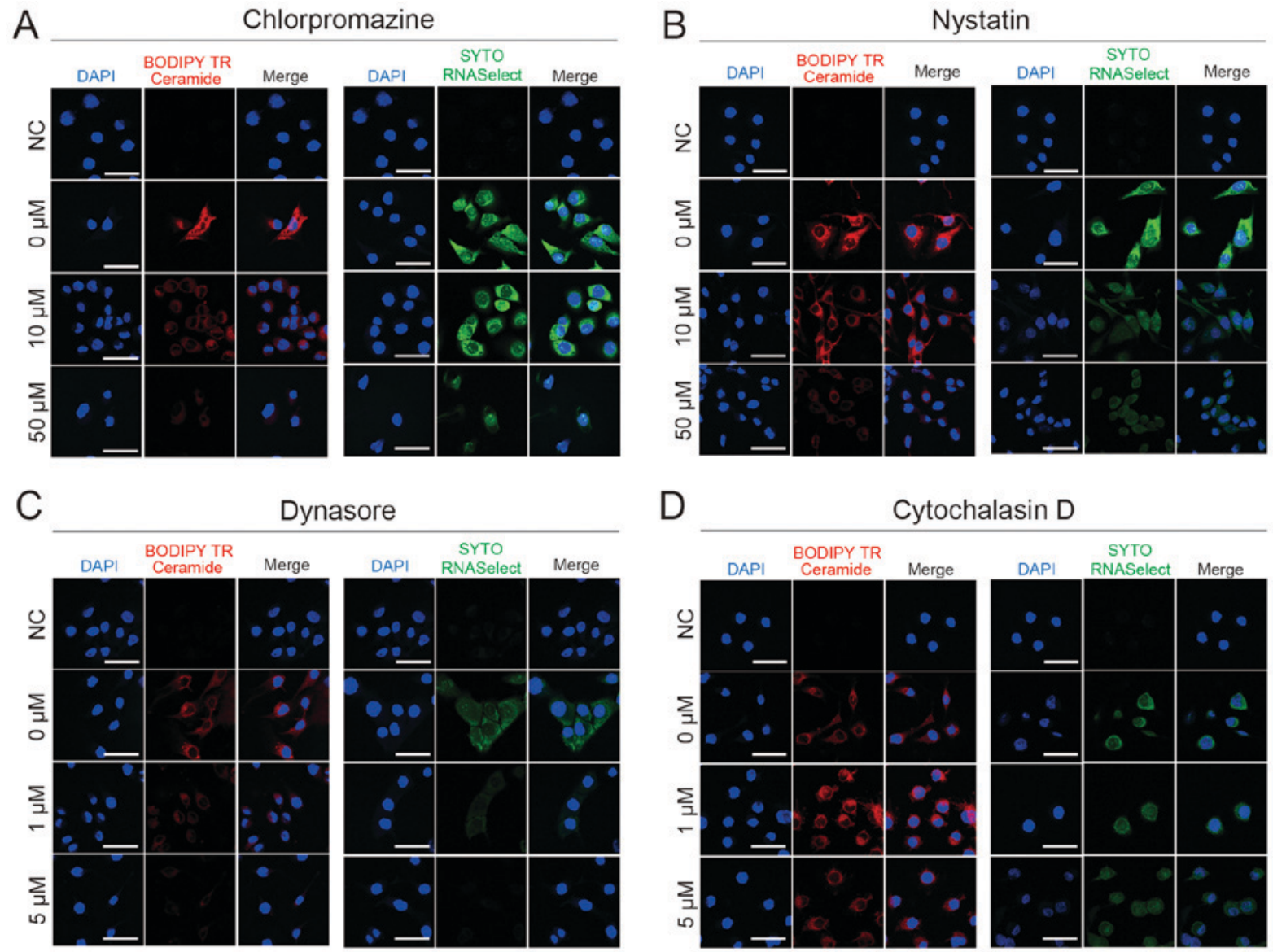

Figure 2. Uptake of EVs released from PK-45H cells by PK-45P cells. EVs released from PK-45H cells were stained using the BODIPY TR Ceramide reagent or SYTO RNA Select reagent (Thermo Fisher Scientific, Inc.). EVs stained with fluorescent dyes were added to culture media of PK-45P cells treated with (A) chlorpromazine, (B) nystatin, (C) dynasore, and (D) cytochalasin D. PK-45P cells were treated with chlorpromazine or nystatin at concentrations of 0, 10, or $50 \mu \mathrm{M}$ and with dynasore or cytochalasin D at concentrations of 0,1 , or $5 \mu \mathrm{M}$. Fluorescence images were photographed using a confocal laser scanning microscope (LSM710; Carl Zeiss). Nuclei were stained using DAPI. Scale bar, $50 \mu \mathrm{m}$. The experiments were performed twice to confirm reproducibility. EV, extracellular vesicle; $\mathrm{NC}$, negative control.

molecules in recipient cells (37). Hence, we used western blotting to assess the phosphorylation status of MAPK-related molecules, such as c-RAF, MEK1/2, ERK1/2, and p90RSK, in PK-45P cells treated with $10 \mathrm{ng} / \mu 1$ of EVs released from PK-45H cells. Additionally, EVs released from PK-45H cells led to an increase in the expression of phospho-c-RAF 

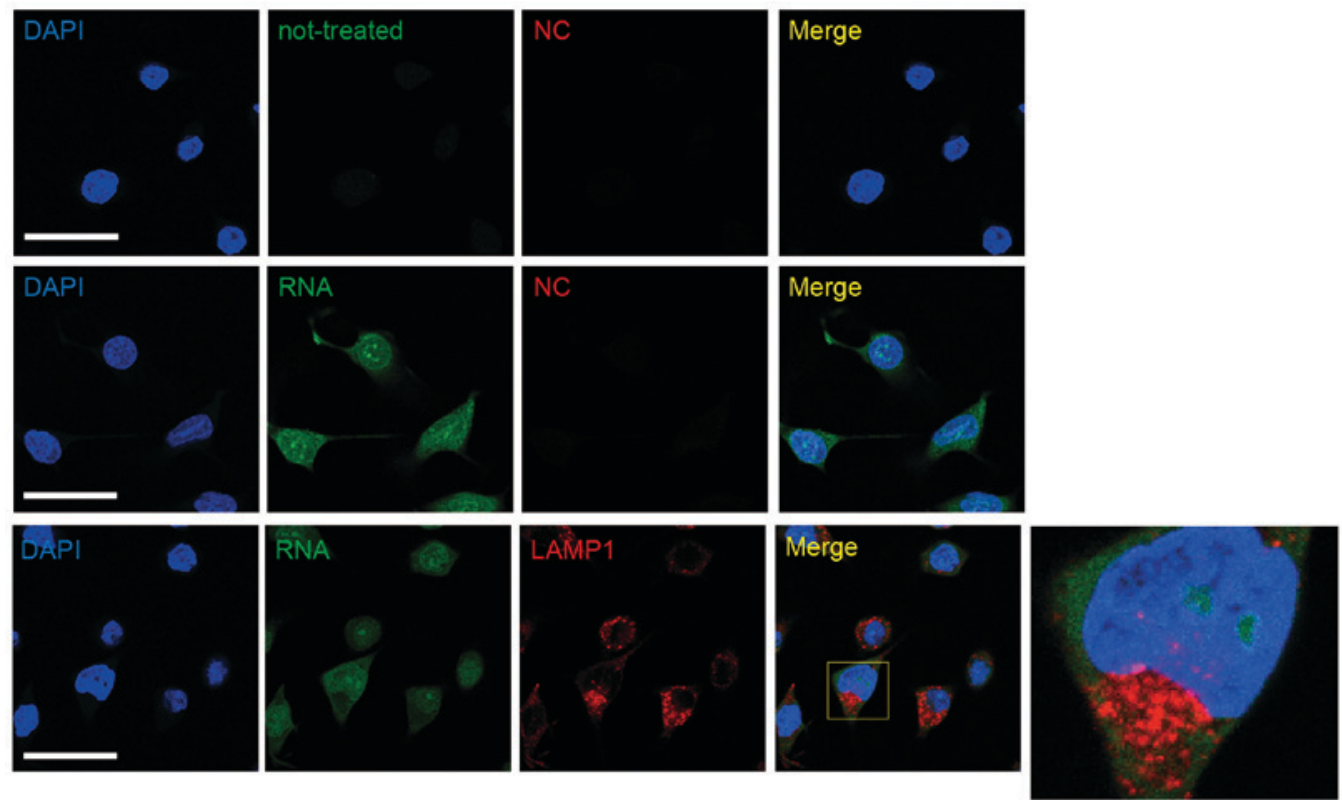

Figure 3. Localization of EV RNAs derived from PK-45H cells into PK-45P cells. EVs released from PK-45H cells were stained using the SYTO RNA Select reagent (Thermo Fisher Scientific, Inc.). EVs stained with fluorescent dyes were added to culture media of PK-45P cells. EV RNAs taken up by PK-45P cells are indicated by green fluorescence. Fluorescence immunostaining of LAMP1 was performed using these PK-45P cells. LAMP1 is indicated by red fluorescence. Fluorescence images were photographed using a confocal laser scanning microscope (LSM710; Carl Zeiss). Nuclei were stained using DAPI. Scale bar, $50 \mu \mathrm{m}$. The experiments were performed twice to confirm reproducibility. EV, extracellular vesicle; $\mathrm{NC}$, negative control.

A

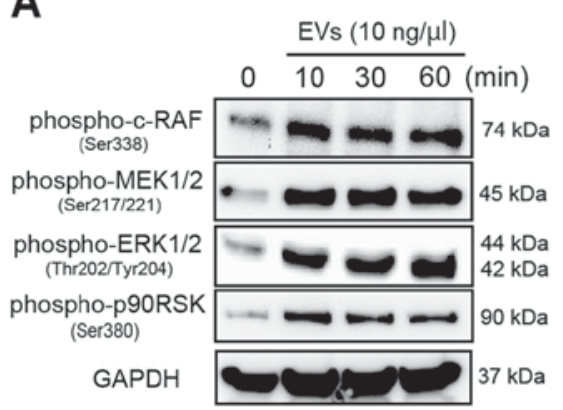

B

EVs $(10 \mathrm{ng} / \mu \mathrm{l})-+-+$

Dynasore $(5 \mu \mathrm{M})--++$

phospho-ERK1/2 $44 \mathrm{kDa}$

(Thr202/Tyr204) $42 \mathrm{kDa}$

Total-ERK $1 / 2=4 \mathrm{kDa}$
C

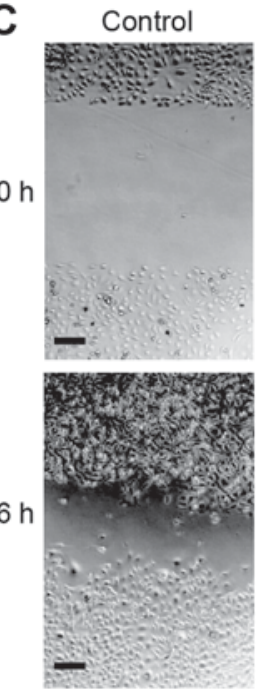

EVs $(10 \mathrm{ng} / \mu \mathrm{l})$

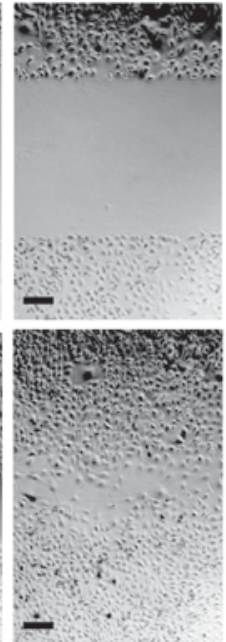

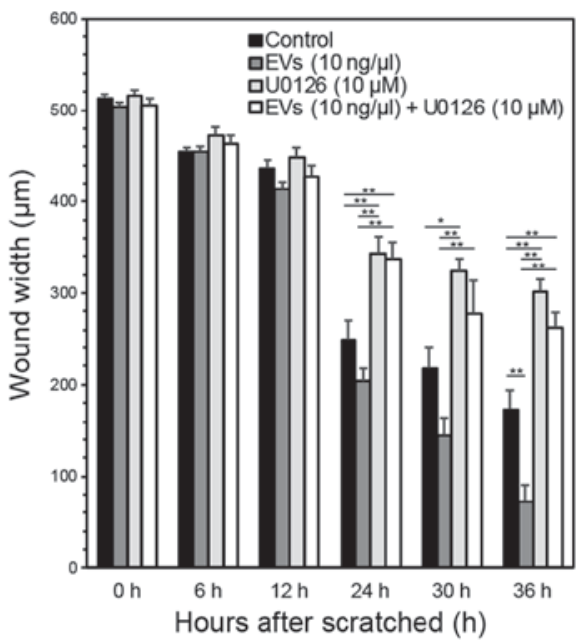

Figure 4. Activation of the classical MAPK pathway and effects on cell migration in EVs-treated PK-45P cells released from PK-45H cells. (A) The phosphorylation status of classical MAPK molecules, c-RAF, MEK1/2, ERK1/2, and p90RSK in EVs-treated PK-45P cells released from PK-45H cells. Phosphorylated proteins were detected using western blotting. PK-45P cells were incubated with EVs released from PK-45H cells for $0,10,30$, or 60 min at $37^{\circ} \mathrm{C}$ in a humidified atmosphere of 5\% $\mathrm{CO}_{2}$. (B) Effects on ERK1/2 activation in EVs-treated PK-45P cells released from PK-45H cells in the presence or absence of $5 \mu \mathrm{M}$ dynasore. Expressions of phospho-ERK1/2 and total-ERK1/2 were examined using western blotting. PK- $45 \mathrm{P}$ cells were incubated for $10 \mathrm{~min}$ at $37^{\circ} \mathrm{C}$ in a humidified atmosphere of $5 \% \mathrm{CO}_{2}$. (C) A photograph of the wound width in PK-45P cells cultures $36 \mathrm{~h}$ after scratching. Scale bar, $200 \mu \mathrm{m}$. (D) Wound width $(\mu \mathrm{m})$ at the indicated time points after scratching PK-45P cells cultures treated with EVs released from PK- $45 \mathrm{H}$ cells at the final concentration of $10 \mathrm{ng} / \mu 1$ for $0,6,12,24$, 30 , or $36 \mathrm{~h}$. Cell migration was assessed in cultures incubated with U0126, MEK1/2 inhibitor, and/or EVs released from PK-45H cells. All bars, mean \pm standard error of the mean. Each 6 sample was used. Statistical comparisons were determined using the one-way ANOVA with Tukey's multiple comparisons. The experiments were performed twice to confirm reproducibility. ${ }^{*} \mathrm{P}<0.05$ and $^{* *} \mathrm{P}<0.01$. MAPK, mitogen-activated protein kinase; EV, extracellular vesicle.

(Ser338), phospho-MEK1/2 (Ser217/221), phospho-ERK1/2 (Thr202/Tyr204), and phospho-p90RSK (Ser380) in PK-45P cells within $10 \mathrm{~min}$ (Fig. 4A), exhibiting that EVs released from PK-45H cells can result in the activation of classical MAPK signaling pathways in PK-45P cells.
We assessed the expression of phospho-ERK1/2 protein in PK-45P cells treated with $10 \mathrm{ng} / \mu \mathrm{l}$ of EVs in the presence or absence of $5 \mu \mathrm{M}$ dynasore to confirm whether the activation of the classical MAPK pathway had been induced by the uptake of EVs released from PK-45H cells. Accordingly, EVs released 
from $\mathrm{PK}-45 \mathrm{H}$ cells induced an increase in the expression of phospho-ERK1/2 (Thr202/Tyr204) in PK-45P cells without dynasore, which was inhibited by treatment with $5 \mu \mathrm{M}$ dynasore (Fig. 4B). This finding suggested that the enhancement of ERK1/2 phosphorylation by EVs among pancreatic cancer cells is dependent on dynamin-related endocytosis.

Reportedly, the activation of the classical MAPK pathway by EVs has been reported to induce cell growth and migration $(38,39)$. We performed a wound-healing assay using culture media in the presence or absence of the MEK1/2 inhibitor U0126 to assess whether the activation of the classical MAPK pathway through EVs released from PK-45H cells altered cell migration in PK-45P cells (Fig. 4C and D). The addition of $10 \mathrm{ng} / \mu \mathrm{l}$ of EVs released from PK-45H cells to culture media of scratched PK-45P cells led significantly higher migration of PK-45P cells for $36 \mathrm{~h}$ compared with that of untreated controls $(\mathrm{P}<0.01)$, suggesting that EVs released from $\mathrm{PK}-45 \mathrm{H}$ cells enhance the migration of PK-45P cells. Additionally, PK-45P cells treated with U0126 for 24, 30, and $36 \mathrm{~h}$ exhibited significantly lower cell migration compared with untreated controls at each time point $(\mathrm{P}<0.01, \mathrm{P}<0.05$, and $\mathrm{P}<0.01$, respectively), suggesting that $\mathrm{PK}-45 \mathrm{P}$ cells induce cell migration through the classical MAPK pathway. Furthermore, U0126- and EV-treated PK-45P cells exhibited significantly lower cell migration after 24, 30, and $36 \mathrm{~h}$ compared with those treated with EV in the absence of U0126 at each time point $(\mathrm{P}<0.01, \mathrm{P}<0.01$, and $\mathrm{P}<0.01$, respectively). These findings suggested that EVs released from $\mathrm{PK}-45 \mathrm{H}$ cells enhance the migration of PK-45P cells primarily through the classical MAPK pathway.

\section{Discussion}

This study demonstrated that EVs released from pancreatic cancer cells enhanced the capability of cell migration in other pancreatic cancer cells. Also, it elucidates that the uptake of EVs through dynamin-related endocytosis induced the activation of the classical MAPK signaling pathway. These findings suggest that pancreatic cancer cells could activate each other through EVs and that EVs could be involved in cancer invasion and transfer in a tumor microenvironment.

A tumor comprises various cells, such as cancer cells, cancer stem cells, normal cells, fibroblast cells, endothelial cells, and immune cells (40). EVs act on target cells by circulating throughout the extracellular space, transferring into neighbor cells within the same environment and taking up contents to exert specific functions (41). To date, the distinct mechanisms through which EVs participate in tumorigenesis and progression through various signaling pathways have been comprehensively investigated (42-44). This study suggests that the uptake of EVs activates the classical MAPK signaling pathway. Yang et al reported that the phosphorylation of ERK1/2 and AKT was enhanced by treatment with EVs released from cancer cells (45). Additionally, research has proven that EVs released from cancer cells carry crucial clinical relevance given their ability to modify the tumor microenvironment, facilitate the epithelial-to-mesenchymal transition and cytoskeleton reorganization, promote tumor angiogenesis, and affect tumor immunity, resulting in invasion and metastasis $(41,46-49)$. In human bladder cells, the treatment of T24 human bladder cancer cells with EVs released from the same tumor cells stimulated the activation of both ERK1/2 and AKT pathways (45). Our findings are consistent with those presented in studies mentioned previously, suggesting that EVs released from pancreatic cancer cells can also activate the classical MAPK signaling pathway in other pancreatic cancer cells under a tumor microenvironment, as well as promote cell migration. On the other hand, MAPK signaling pathway is involved in cell growth. We examined the cell growth in EVs-treated PK-45P cells released from PK-45H cells using the alamarBlue reagent (Thermo Fisher Scientific, Inc.) and found that the capacity of cell growth did not considerably differ between the EV-treatment and EV-nontreatment groups (data not shown). Hence, we believe that cell growth is not induced by EV treatment.

Seemingly, cells uptake EVs through various endocytic pathways, including clathrin-mediated endocytosis, caveolin-mediated endocytosis, macropinocytosis, phagocytosis, and lipid raft-mediated internalization (50). Several studies have reported that incubating cells at $4^{\circ} \mathrm{C}$ remarkably decreases their capacity to internalize EVs, suggesting that the uptake is a process requiring energy, such as clathrin-mediated endocytosis, caveolin-mediated endocytosis, and macropinocytosis $(51,52)$. This study suggests that EVs released from pancreatic cancer cells were taken up by other pancreatic cancer cells via dynamin-related endocytosis. Dynamin is a GTPase protein that is essential for membrane fission during clathrin-mediated endocytosis in eukaryotic cells (53). Dynasore is a small, non-selective inhibitor of dynamin GTPase activity (54). Previously, dynasore has been reported to inhibit the uptake of EVs $(55,56)$. The findings of studies mentioned above are consistent with this study. Remarkably, EVs have been reported to enhance the AKT phosphorylation and cell migration, both of which had been inhibited by dynasore (57). Thus, the inhibition of dynamin might suppress the AKT phosphorylation and cell migration in EVs-treated PK-45P cells released from PK-45H cells.

Additionally, dynamin is involved in caveolin-mediated endocytosis $(58,59)$. Caveolin is a protein that is required and sufficient for the formation of caveolae (60), specialized domains of the plasma membrane that are implicated in the sequestration of various lipid and protein molecules (61). Nanbo et al reported that specific knockdown of the caveolin-1 (CAV1) gene results in decreased caveolin-1 protein and considerably impaired the EV uptake (62). Likewise, this study demonstrated that the uptake of EVs among pancreatic cancer cells was suppressed by the treatment with nystatin, which works as a caveolin-mediated endocytosis inhibitor. However, Svensson et al reported that caveolin-1 protein negatively regulated the uptake of EVs by glioblastoma cells (63). Hence, further investigation is warranted to elucidate the uptake of EVs through caveolin-mediated endocytosis.

Macropinocytosis is an endocytic uptake pathway that involves the formation of invaginated membrane ruffles that then pinch off into the intracellular compartment (64). Additionally, dynamin is not involved in the uptake through macropinocytosis. Cytochalasin D is a metabolite that depolymerizes the actin filament network, causing the inhibition of macropinocytosis (65). This study demonstrates that EVs released from $\mathrm{PK}-45 \mathrm{H}$ cells were not taken up by PK-45P 
cells through micropinocytosis. Thus, we believe that EVs released from PK-45H cells were taken up by PK-45P cells via dynamin-related endocytosis. However, one implication of the frequent failure to completely abrogate internalization following treatment with any given inhibitor is that the EV uptake occurs through more than one mechanism $(23,66)$. In fact, it seems likely that a heterogeneous population of EVs may gain entry into a cell by more than one route. Hence, further studies are warranted regarding the specific uptake of EVs in drug delivery systems.

In this study, it is difficult to examine the effects on specific EVs released from PK-45H cells, such as exosomes, shedding microvesicles or apoptotic bodies, in PK-45P cells, because EVs were collected by ultracentrifugation. Therefore, it is not clear what type of EV has the most effect on PK-45P cells. In future, we will perform the selection of EV type using gravity separation and specific markers of each EV released from cancer cells of high metastasis and examine what type of EV exerts the biggest effect on cancer cells of low metastasis.

\section{Acknowledgements}

Not applicable.

\section{Funding}

This study was supported, in part, by a Hirosaki University Institutional Research Grant for Young Scientists.

\section{Availability of data and materials}

The datasets used and/or analyzed during the current study are available from the corresponding author on reasonable request.

\section{Authors' contributions}

$\mathrm{MC}$ was a major contributor in performing experiments and writing the manuscript. SK, AS and SM performed experiments. MC supervised this study. All authors approved the final manuscript.

\section{Ethics approval and consent to participate}

Not applicable.

\section{Patient consent for publication}

Not applicable.

\section{Competing interests}

The authors declare that they have no competing interests.

\section{References}

1. Raposo G and Stoorvogel W: Extracellular vesicles: Exosomes, microvesicles, and friends. J Cell Biol 200: 373-383, 2013.

2. Pan BT and Johnstone RM: Fate of the transferrin receptor during maturation of sheep reticulocytes in vitro: Selective externalization of the receptor. Cell 33: 967-978, 1983.
3. Skog J, Wurdinger T, van Rijn S, Meijer DH, Gainche L, Curry WT Jr, Carter BS, Krichevsky AM and Breakefield XO: Glioblastoma microvesicles transport RNA and proteins that promote tumour growth and provide diagnostic biomarkers. Nat Cell Biol 10: 1470-1476, 2008.

4. Al-Nedawi K, Meehan B, Micallef J, Lhotak V, May L, Guha A and Rak J: Intercellular transfer of the oncogenic receptor EGFRvIII by microvesicles derived from tumour cells. Nat Cell Biol 10: 619-624, 2008.

5. Antonyak MA, Li B, Boroughs LK, Johnson JL, Druso JE, Bryant KL, Holowka DA and Cerione RA: Cancer cell-derived microvesicles induce transformation by transferring tissue transglutaminase and fibronectin to recipient cells. Proc Natl Acad Sci USA 108: 4852-4857, 2011.

6. Valadi H, Ekström K, Bossios A, Sjöstrand M, Lee JJ and Lötvall JO: Exosome-mediated transfer of mRNAs and microRNAs is a novel mechanism of genetic exchange between cells. Nat Cell Biol 9: 654-659, 2007.

7. Montecalvo A,Larregina AT, Shufesky WJ, Stolz DB, Sullivan ML, Karlsson JM, Baty CJ, Gibson GA, Erdos G, Wang Z, et al: Mechanism of transfer of functional microRNAs between mouse dendritic cells via exosomes. Blood 119: 756-766, 2012.

8. Fabbri M, Paone A, Calore F, Galli R, Gaudio E, Santhanam R, Lovat F, Fadda P, Mao C, Nuovo GJ, et al: MicroRNAs bind to Toll-like receptors to induce prometastatic inflammatory response. Proc Natl Acad Sci USA 109: E2110-E2116, 2012.

9. Qu L, Ding J, Chen C, Wu ZJ, Liu B, Gao Y, Chen W, Liu F, Sun W, Li XF, et al: Exosome-transmitted lncARSR promotes sunitinib resistance in renal cancer by acting as a competing endogenous RNA. Cancer Cell 29: 653-668, 2016.

10. Chiba M, Kimura M and Asari S: Exosomes secreted from human colorectal cancer cell lines contain mRNAs, microRNAs and natural antisense RNAs, that can transfer into the human hepatoma HepG2 and lung cancer A549 cell lines. Oncol Rep 28: 1551-1558, 2012.

11. Balaj L, Lessard R, Dai L, Cho YJ, Pomeroy SL, Breakefield XO and Skog J: Tumour microvesicles contain retrotransposon elements and amplified oncogene sequences. Nat Commun 2: 180,2011

12. Frühbeis C, Fröhlich D and Krämer-Albers EM: Emerging roles of exosomes in neuron-glia communication. Front Physiol 3: 119, 2012.

13. Marcilla A, Trelis M, Cortés A, Sotillo J, Cantalapiedra F, Minguez MT, Valero ML, Sánchez del Pino MM, Muñoz-Antoli C, Toledo R and Bernal D: Extracellular vesicles from parasitic helminths contain specific excretory/secretory proteins and are internalized in intestinal host cells. PLoS One 7: e45974, 2012.

14. Regev-Rudzki N, Wilson DW, Carvalho TG, Sisquella X, Coleman BM, Rug M, Bursac D, Angrisano F, Gee M, Hill AF, et al: Cell-cell communication between malaria-infected red blood cells via exosome-like vesicles. Cell 153: 1120-1133, 2013.

15. Mathivanan S, Ji H and Simpson RJ: Exosomes: Extracellular organelles important in intercellular communication. J Proteomics 73: 1907-1920, 2010.

16. Camussi G, Deregibus MC, Bruno S, Cantaluppi V and Biancone L: Exosomes/microvesicles as a mechanism of cell-to-cell communication. Kidney Int 78: 838-848, 2010.

17. Christianson HC, Svensson KJ and Belting M: Exosome and microvesicle mediated phene transfer in mammalian cells. Semin Cancer Biol 28: 31-38, 2014.

18. Katzmann DJ, Babst M and Emr SD: Ubiquitin-dependent sorting into the multivesicular body pathway requires the function of a conserved endosomal protein sorting complex, ESCRT-I. Cell 106: 145-155, 2001

19. Kowal J, Tkach M and Théry C: Biogenesis and secretion of exosomes. Curr Opin Cell Biol 29: 116-125, 2014.

20. Kosaka N, Iguchi H, Yoshioka Y, Takeshita F, Matsuki Y and Ochiya T: Secretory mechanisms and intercellular transfer of microRNAs in living cells. J Biol Chem 285: 17442-17452, 2010.

21. Kosaka N, Iguchi H, Hagiwara K, Yoshioka Y, Takeshita F and Ochiya T: Neutral sphingomyelinase 2 (nSMase2)-dependent exosomal transfer of angiogenic microRNAs regulate cancer cell metastasis. J Biol Chem 288: 10849-10859, 2013.

22. Mulcahy LA, Pink RC and Carter DR: Routes and mechanisms of extracellular vesicle uptake. J Extracell Vesicles 3, 2014.

23. Feng D, Zhao WL, Ye YY, Bai XC, Liu RQ, Chang LF, Zhou Q and Sui SF: Cellular internalization of exosomes occurs through phagocytosis. Traffic 11: 675-687, 2010. 
24. Fitzner D, Schnaars M, van Rossum D, Krishnamoorthy G, Dibaj P, Bakhti M, Regen T, Hanisch UK and Simons M: Selective transfer of exosomes from oligodendrocytes to microglia by macropinocytosis. J Cell Sci 124: 447-458, 2011.

25. Tian T, Zhu YL, Zhou YY, Liang GF, Wang YY, Hu FH and Xiao ZD: Exosome uptake through clathrin-mediated endocytosis and macropinocytosis and mediating miR-21 delivery. J Biol Chem 289: 22258-22267, 2014.

26. Siegel RL, Miller KD and Jemal A: Cancer statistics, 2018. CA Cancer J Clin 68: 7-30, 2018

27. Stathis A and Moore MJ: Advanced pancreatic carcinoma: Current treatment and future challenges. Nat Rev Clin Oncol 7: $163-172,2010$

28. Provenzano PP, Cuevas C, Chang AE, Goel VK, Von Hoff DD and Hingorani SR: Enzymatic targeting of the stroma ablates physical barriers to treatment of pancreatic ductal adenocarcinoma. Cancer Cell 21: 418-429, 2012.

29. Wolfgang CL, Herman JM, Laheru DA, Klein AP, Erdek MA, Fishman EK and Hruban RH: Recent progress in pancreatic cancer. CA Cancer J Clin 63: 318-348, 2013.

30. Nazarenko I, Rana S, Baumann A, McAlear J, Hellwig A Trendelenburg M, Lochnit G, Preissner KT and Zöller M: Cell surface tetraspanin Tspan8 contributes to molecular pathways of exosome-induced endothelial cell activation. Cancer Res 70 $1668-1678,2010$

31. Masamune A, Yoshida N, Hamada S, Takikawa T, Nabeshima T and Shimosegawa T: Exosomes derived from pancreatic cancer cells induce activation and profibrogenic activities in pancreatic stellate cells. Biochem Biophys Res Commun 495: 71-77, 2018

32. Pang W, Su J, Wang Y, Feng H, Dai X, Yuan Y, Chen X and Yao W: Pancreatic cancer-secreted miR-155 implicates in the conversion from normal fibroblasts to cancer-associated fibroblasts. Cancer Sci 106: 1362-1369, 2015.

33. Ding G, Zhou L, Qian Y, Fu M, Chen J, Chen J, Xiang J, Wu Z, Jiang $G$ and Cao L: Pancreatic cancer-derived exosomes transfer miRNAs to dendritic cells and inhibit RFXAP expression via miR-212-3p. Oncotarget 6: 29877-29888, 2015

34. Su MJ, Aldawsari $\mathrm{H}$ and Amiji M: Pancreatic cancer cell exosome-mediated macrophage reprogramming and the role of microRNAs 155 and $125 \mathrm{~b} 2$ transfection using nanoparticle delivery systems. Sci Rep 6: 30110, 2016.

35. Amikura K, Kobori M and Matsuno S: The mechanism of liver metastasis in pancreatic cancer. Jpn J Gastroenterol Surg 24: 1112-1116, 1991

36. French KC, Antonyak MA and Cerione RA: Extracellular vesicle docking at the cellular port: Extracellular vesicle binding and uptake. Semin Cell Dev Biol 67: 48-55, 2017.

37. Qu JL, Qu XJ, Zhao MF, Teng YE, Zhang Y, Hou KZ, Jiang YH, Yang XH and Liu YP: Gastric cancer exosomes promote tumou cell proliferation through PI3K/Akt and MAPK/ERK activation. Dig Liver Dis 41: 875-880, 2009.

38. Zhu W, Huang L, Li Y, Zhang X, Gu J, Yan Y, Xu X, Wang M, Qian $\mathrm{H}$ and $\mathrm{Xu} \mathrm{W}$ : Exosomes derived from human bone marrow mesenchymal stem cells promote tumor growth in vivo. Cancer Lett 315: 28-37, 2012

39. Yoon YJ, Kim DK, Yoon CM, Park J, Kim YK, Roh TY and Gho YS: Egr-1 activation by cancer-derived extracellular vesicles promotes endothelial cell migration via ERK1/2 and JNK signaling pathways. PLoS One 9: e115170, 2014.

40. Pietras A: Cancer stem cells in tumor heterogeneity. Adv Cancer Res 112: 255-281, 2011

41. Maia J, Caja S, Strano Moraes MC, Couto N and Costa-Silva B: Exosome-based cell-cell communication in the tumor microenvironment. Front Cell Dev Biol 6: 18, 2018.

42. Whiteside TL: Tumor-derived exosomes and their role in cancer progression. Adv Clin Chem 74: 103-141, 2016

43. Kanada M, Bachmann $\mathrm{MH}$ and Contag $\mathrm{CH}$ : Signaling by extracellular vesicles advances cancer hallmarks. Trends Cancer 2 84-94, 2016.

44. Gangoda L, Boukouris S, Liem M, Kalra H and Mathivanan S: Extracellular vesicles including exosomes are mediators of signal transduction: Are they protective or pathogenic? Proteomics 15: 260-271, 2015

45. Yang L, Wu XH, Wang D, Luo CL and Chen LX: Bladder cancer cell-derived exosomes inhibit tumor cell apoptosis and induce cell proliferation in vitro. Mol Med Rep 8: 1272-1278, 2013.
46. Li W, Zhang X, Wang J, Li M, Cao C, Tan J, Ma D and Gao Q: TGF $\beta 1$ in fibroblasts-derived exosomes promotes epithelial-mesenchymal transition of ovarian cancer cells. Oncotarget 8: 96035-96047, 2017.

47. Chen Z, Yang L, Cui Y, Zhou Y, Yin X, Guo J, Zhang G, Wang T and He QY: Cytoskeleton-centric protein transportation by exosomes transforms tumor-favorable macrophages. Oncotarget 7: 67387-67402, 2016.

48. Lee HY, Chen CK, Ho CM, Lee SS, Chang CY, Chen KJ and Jou YS: EIF3C-enhanced exosome secretion promotes angiogenesis and tumorigenesis of human hepatocellular carcinoma. Oncotarget 9: 13193-13205, 2018

49. Chen X, Ying X, Wang X, Wu X, Zhu Q and Wang X: Exosomes derived from hypoxic epithelial ovarian cancer deliver microRNA-940 to induce macrophage M2 polarization. Oncol Rep 38: 522-528, 2017.

50. McKelvey KJ, Powell KL, Ashton AW, Morris JM and McCracken SA: Exosomes: Mechanisms of uptake. J Circ Biomark 4: 7, 2015.

51. Escrevente C, Keller S, Altevogt $\mathrm{P}$ and Costa J: Interaction and uptake of exosomes by ovarian cancer cells. BMC Cancer 11: 108,2011

52. Morelli AE, Larregina AT, Shufesky WJ, Sullivan ML, Stolz DB Papworth GD, Zahorchak AF, Logar AJ, Wang Z, Watkins SC, et al: Endocytosis, intracellular sorting, and processing of exosomes by dendritic cells. Blood 104: 3257-3266, 2004

53. Singh M, Jadhav HR and Bhatt T: Dynamin functions and ligands: Classical mechanisms behind. Mol Pharmacol 91: 123-134, 2017.

54. Macia E, Ehrlich M, Massol R, Boucrot E, Brunner C and Kirchhausen T: Dynasore, a cell-permeable inhibitor of dynamin. Dev Cell 10: 839-850, 2006.

55. Kawamoto T, Ohga N, Akiyama K, Hirata N, Kitahara S, Maishi N, Osawa T, Yamamoto K, Kondoh M, Shindoh M, et al: Tumor-derived microvesicles induce proangiogenic phenotype in endothelial cells via endocytosis. PLoS One 7: e34045, 2012.

56. Roberts-Dalton HD, Cocks A, Falcon-Perez JM, Sayers EJ, Webber JP, Watson P, Clayton A and Jones AT: Fluorescence labelling of extracellular vesicles using a novel thiol-based strategy for quantitative analysis of cellular delivery and intracellular traffic. Nanoscale 9: 13693-13706, 2017

57. Wang R, Ding Q, Yaqoob U, de Assuncao TM, Verma VK, Hirsova P, Cao S, Mukhopadhyay D, Huebert RC and Shah VH: Exosome adherence and internalization by hepatic stellate cells triggers sphingosine 1-phosphate-dependent migration. J Biol Chem 290: 30684-30696, 2015.

58. Lajoie P and Nabi IR: Lipid rafts, caveolae, and their endocytosis. Int Rev Cell Mol Biol 282: 135-163, 2010.

59. Bourseau-Guilmain E, Menard JA, Lindqvist E, Indira Chandran V, Christianson HC, Magaña MC, Lidfeldt J, Marko-Varga G, Welinder C and Belting M: Hypoxia regulates global membrane protein endocytosis through caveolin-1 in cancer cells. Nat Commun 7: 11371, 2016.

60. Jin X, Newton JR, Montgomery-Smith S and Smith G: A generalized kinetic model for amine modification of proteins with application to phage display. Biotechniques 46: 175-182, 2009.

61. Gu X, Reagan AM, McClellan ME and Elliott MH: Caveolins and caveolae in ocular physiology and pathophysiology. Prog Retin Eye Res 56: 84-106, 2017.

62. Nanbo A, Kawanishi E, Yoshida R and Yoshiyama H: Exosomes derived from Epstein-Barr virus-infected cells are internalized via caveola-dependent endocytosis and promote phenotypic modulation in target cells. J Virol 87: 10334-10347, 2013.

63. Svensson KJ, Christianson HC, Wittrup A, Bourseau-Guilmain E, Lindqvist E, Svensson LM, Mörgelin M and Belting M: Exosome uptake depends on ERK1/2-heat shock protein 27 signaling and lipid Raft-mediated endocytosis negatively regulated by caveolin-1. J Biol Chem 288: 17713-17724, 2013.

64. Ha KD, Bidlingmaier SM and Liu B: Macropinocytosis exploitation by cancers and cancer therapeutics. Front Physiol 7: 381, 2016.

65. Lamaze C, Fujimoto LM, Yin HL and Schmid SL: The actin cytoskeleton is required for receptor-mediated endocytosis in mammalian cells. J Biol Chem 272: 20332-20335, 1997.

66. Barrès C, Blanc L, Bette-Bobillo P, André S, Mamoun R, Gabius HJ and Vidal M: Galectin-5 is bound onto the surface of rat reticulocyte exosomes and modulates vesicle uptake by macrophages. Blood 115: 696-705, 2010 